\title{
$\underline{\text { A Re-Interpretation of the Normative Foundations of Majority Rule }}{ }^{1}$ Introduction
}

In contemporary democratic theory, majority rule holds a special place among group decision making rules on two alternatives. As Robert Dahl put it, substantive debate tends not to focus on whether majority rule is a necessary condition for democracy, but whether it is sufficient (Dahl 1989, 135). Mathias Risse noted that when there are multiple alternatives, there is the tendency to assume that the normative desirability of majority rule generalizes to multiple alternatives, though such generalizations are often questionable (Risse 2004). The most common normative generalization of majority rule is called the Condorcet criterion. We will later give a more formal definition of Condorcet methods (i.e. voting systems that satisfy this criterion), but we give a brief description here.

Roughly speaking, a voting system (or more technically speaking, a social welfare function) consists of two procedures: balloting and aggregation (Merrill III and Nagel 1987). Typically in the literature, the balloting procedure of voting systems is ignored and left implicit (Goodin and List 2006). But balloting procedures are important; otherwise, for example, plurality voting, as usually defined, ${ }^{2}$ would be classified as a Condorcet method. That would be a classification that is generally rejected.

\footnotetext{
${ }^{1}$ Author Information: Mahendra Prasad, Department of Political Science, UC Berkeley, Email: mrprasad@berkeley.edu
}

${ }^{2}$ A conventional definition of plurality voting assumes voters submit a ballot with one alternative ranked above all others, with the remaining alternatives ranked equally. The only other kind of ballot permitted is when the voter expresses indifference between all alternatives (Goodin and List 2006). 
If there are $n$ alternatives under consideration, then the balloting procedure of a Condorcet method must allow each voter the opportunity to express any of the $n$ ! logically possible preference orders (that are strict, complete, and transitive) on their ballot. ${ }^{3}$ The aggregation procedure of a Condorcet method requires that if there exists an alternative, say $a_{x}$, which beats all other alternatives under consideration one-on-one via majority rule, then such an $a_{x}$ should be socially ranked above all other alternatives under consideration.

What I seek to show in this piece is that another Arrovian voting system, ${ }^{4}$ which we will call unrestricted non-polychotomous voting (UNV), does a better job of generalizing normative arguments for majority rule than do other Arrovian voting systems. That said, one issue that is beyond the scope of this piece is the concern that voters may misrepresent their preferences on their ballots to game the outcome of the election. This is a concern which is endemic to virtually all voting systems, as demonstrated by the Gibbard-Satterthwaite theorem and related results (A. Taylor 2005). Those results have encouraged the development of the mechanism design literature, where a designer with particular norms in mind, seeks to construct a decision making mechanism that achieves such norms given that players (e.g. voters) are strategic. Our focus is on the designer's norms themselves. When voters are not trying to misrepresent their preferences to

\footnotetext{
${ }^{3}$ We define strict, complete, transitive, and preference order in more detail later, but for now we will give this less formal definition of strict complete transitive preference order. A strict complete transitive preference order ranks exactly one alternative in first place, exactly one alternative in second place, and so forth such that all $n$ alternatives are ranked.

${ }^{4}$ The Arrovian framework is the framework that Kenneth Arrow laid out for voting systems in his landmark book Social Choice and Individual Values. Arrovian voting systems are voting systems that fall within that framework (Arrow 1963).
} 
game the outcome, voting systems can be understood as representing norms. For example, assuming its balloting procedure, plurality voting can be understood as representing a norm that the group decision should maximize the number of voters who get their first preference implemented. What we are trying to do here is give arguments as to why a mechanism designer might seek norms represented by UNV, rather than the norms represented by Condorcet methods. As such, we leave issues of ballot misrepresentation aside for mechanism designers. ${ }^{5}$

UNV can be informally defined as follows: Specifically, a voter chooses some non-empty subset of the alternatives under consideration; call this her choice set, while the remaining alternatives, if any, are her non-choice set. The voter is indifferent between any two alternatives within her choice set; she is also indifferent between any two alternatives in her non-choice set. However, she prefers any alternative in her choice set over any alternative in her non-choice set. The aggregation procedure of UNV counts the number of voters that place an alternative, say $a_{x}$, in their respective choice sets. If $a_{x}$ occurs in more choice sets than say $a_{y}$, then $a_{x}$ is socially ranked above $a_{y}$. If $a_{x}$ and $a_{y}$ occur in the same number of choice sets, then UNV socially ranks $a_{x}$ and $a_{y}$ equally.

It is important to distinguish approval voting from UNV. With approval voting, a voter marks each alternative she approves of; alternatives are socially rank ordered by the number of approvals they receive (Brams and Fishburn 2007). But it is important to note, approval voting, as typically defined, is a non-Arrovian voting system, as it uses information beyond voter preference orders as inputs. For example, a voter may prefer $a_{x}$ over $a_{y}$, but approve both. Meanwhile UNV is clearly an Arrovian voting system, where ballots are preference orders and the output is

\footnotetext{
${ }^{5}$ There is evidence to suggest that in elections with large numbers of voters, voters tend to be sincere (Feddersen, Gailmard and Sandroni 2009).
} 
a preference order. This is important because we are going to show that among Arrovian voting systems, UNV better generalizes majority rule to multiple alternatives than do any other Arrovian voting system.

In his 1989 classic, Democracy and Its Critics, Robert Dahl put forth four arguments he believed were the four best arguments for why majority rule is the optimal democratic group decision rule for two alternatives (Dahl 1989, 136-137). Specifically, we will argue that UNV generalizes those four arguments as good as or better than do Condorcet methods or any other Arrovian voting systems generalize those four arguments to multiple alternatives.

We proceed in the following fashion. First, we will present some basic definitions necessary for our discussion. Second, we will proceed through each of Dahl's four arguments for majority rule and summarize why UNV generalizes these arguments to multiple alternatives as good as or better than other Arrovian voting systems. Third, we deal with a main rebuttal of UNV, that it overly restricts voters' ballots, by discussing three different general areas of democratic decision making where UNV is not overly restrictive. Fourth, roughly speaking, we show that in areas where the UNV balloting procedure is appropriate, William Riker's contention (that populist democracy is unintelligible due to the meaninglessness of the aggregation of individual opinions into a social opinion, because of Arrow's theorem) is considerably weakened because we can show that when it is legitimate to appropriately weaken unrestricted domain, UNV can uniquely satisfy much stronger versions of the remaining conditions of Arrow's theorem. Finally, in the Supporting Information section, we provide proofs for theorems asserted in the paper. However, the reader should read the Basic Definitions section prior to reading the Supporting Information.

\section{Basic Definitions}


Let $\boldsymbol{A}$ be a finite set of mutually exclusive alternatives, and let $A$ be some finite subset of $n$ mutually exclusive alternatives from $\boldsymbol{A}$ where $n>1$. Let $\boldsymbol{V}$ be a finite set of mutually exclusive voters, and let $V$ be a finite subset of $m$ mutually exclusive voters from $V$ where $m>1$. In accordance with the Arrovian framework, each voter's ballot is a complete and transitive preference order of the $n$ alternatives. A preference order is complete in the sense that for any two alternatives in $A$, say $a_{x}$ and $a_{y}$, exactly one of three relations is true: $a_{x}>a_{y}$ (i.e. $a_{x}$ is ranked above $\left.a_{y}\right), a_{y}>a_{x}$ (i.e. $a_{y}$ is ranked above $a_{x}$ ), or $a_{x} \sim a_{y}$ (i.e. $a_{x}$ and $a_{y}$ are ranked equally). A preference order is transitive in the sense that for any alternatives $a_{x}, a_{y}$, and $a_{z}$ in $A$ it is the case that: ([ $\left(a_{x}>\right.$ $\left.a_{y}\right)$ and $\left.\left(a_{y}>a_{z}\right)\right]$ implies $\left.a_{x}>a_{z}\right),\left(\left[\left(a_{x}>a_{y}\right)\right.\right.$ and $\left.\left(a_{y} \sim a_{z}\right)\right]$ implies $\left.a_{x}>a_{z}\right),\left(\left[\left(a_{x} \sim a_{y}\right)\right.\right.$ and $\left(a_{y}>\right.$ $\left.\left.a_{z}\right)\right]$ implies $\left.a_{x}>a_{z}\right)$, and $\left(\left[\left(a_{x} \sim a_{y}\right)\right.\right.$ and $\left.\left(a_{y} \sim a_{z}\right)\right]$ implies $\left.a_{x} \sim a_{z}\right)$. For our purposes, it is assumed that all preference orders are transitive and complete.

If in a preference order, $a_{x} \sim a_{z}$, then $a_{x}$ and $a_{y}$ belong to the same part of that preference order. All alternatives that are equally ranked with each other within a preference order will be expressed within the same parentheses when expressing that preference order. For example, if in a preference order, $a_{x}, a_{y}$, and $a_{z}$ are ranked equally and no other alternatives in $A$ are ranked equally with them in that preference order, then their part in the preference order will be expressed as $\left(a_{x} a_{y} a_{z}\right)$. Note, if for example, no alternative is equally ranked with say $a_{w}$ in a given preference order, then its part in the preference order is expressed as $\left(a_{w}\right)$. Now, if say $a_{w}>a_{x}$ in a given preference order, then the part to which $a_{w}$ belongs will be expressed to the left of the part to which $a_{x}$ belongs in that given preference order. For example, $\left(a_{w}\right)\left(a_{x} a_{y} a_{z}\right)$ means " $a_{w}$ is ranked above $a_{x}$ and $a_{y}$ and $a_{z}$, while $a_{x}$ and $a_{y}$ and $a_{z}$ are ranked equally".

Note, each preference order consists of a part or parts. For example, consider the preference order $\left(a_{w}\right)\left(a_{x} a_{y}\right)\left(a_{z}\right)$. That preference order consists of three parts, where $\left(a_{w}\right)$ is the first 
part, $\left(a_{x} a_{y}\right)$ is the second part, and $\left(a_{z}\right)$ is the third part. Note, $\left(a_{w}\right)$ is called the first part because there are zero parts ranked above it, $\left(a_{x} a_{y}\right)$ is the second part because there is exactly one part ranked above it, and so forth.

A ballot that contains exactly one part is called a trivial ballot. A ballot that contains exactly two parts is a dichotomous ballot. A ballot that contains more than two parts is called a polychotomous ballot. A collection of $m$ ballots, one from each voter, is a profile.

\section{$\underline{\text { Social Welfare Functions and Voting Systems }}$}

A social welfare function (SWF), takes a profile as its input and outputs a preference order of $A .^{6}$ To avoid confusion between inputted ballots and the outputted preference order, we will refer to preference orders outputted by SWFs as social rank orderings, or simply rank orderings.

As noted before, a SWF can be understood as consisting of two aspects: a balloting procedure and an aggregation procedure. The balloting procedure of a SWF specifies which ballots are available to voters. Thus for example, if there are $c$ number of ballots allowed by a balloting procedure for a particular SWF and the SWF is a total function, then for each of the $c^{m}$ logically possible profiles, the SWF assigns a rank ordering. If the SWF is a partial function, then there exist some profile(s) among the $c^{m}$ profiles for which the SWF does not output a rank ordering. The profile domain of an SWF is the subset of the $c^{m}$ profiles for which the SWF outputs a rank

\footnotetext{
${ }^{6}$ While in theory, SWFs could be used to decide the members of a committee; it seems more normatively appropriate to conceptualize and use the rank ordering of alternatives produced by SWFs as an order of implementation. That is, $a_{x}$ should only be considered for implementation if all other alternatives ranked above it in the social rank ordering are not able to be implemented.
} 
ordering. The aggregation procedure specifies the rank ordering outputted for each profile in the profile domain of an SWF.

There are a few balloting procedures we need to clarify, as they will be used later to describe SWFs. The unrestricted balloting procedure contains all logically possible preference orders of the $n$ alternatives. The strict balloting procedure contains all preference orders on the $n$ alternatives with exactly $n$ parts, but no other preference orders. The UNV balloting procedure contains every non-polychotomous preference order on the $n$ alternatives, and no other preference orders. The trivial balloting procedure allows only the trivial preference order.

Among aggregation procedures, the one of most importance to us is the majority rule aggregation procedure, or majority aggregation procedure for short. Consider any two alternatives from $A$, say $a_{x}$ and $a_{y}$. Suppose $p$ is some profile in the profile domain of a given SWF. If the SWF uses the majority aggregation procedure, then for any such $a_{x}$ and $a_{y}$ and $p$, the following three rules apply: (1) If more voters in $p$ hold $a_{x}>a_{y}$ than hold $a_{y}>a_{x}$, then the SWF must socially rank $a_{x}$ above $a_{y}$ for $p$. (2) If an equal number of voters in $p$ hold $a_{x}>a_{y}$ as hold $a_{y}>a_{x}$, then the SWF must socially rank $a_{x}$ and $a_{y}$ equally for $p$. (3) If more voters in $p$ hold $a_{y}>a_{x}$ than hold $a_{x}>a_{y}$, then the SWF must socially rank $a_{y}$ above $a_{x}$ for $p$.

We now proceed to define some SWFs.

Majority Rule SWF: Technically speaking, this SWF is undefined for elections where $n>2 .{ }^{7}$ The majority rule SWF, where $n=2$, uses the unrestricted balloting procedure. It uses the majority aggregation procedure.

\footnotetext{
${ }^{7}$ There are many SWFs that reduce to the majority rule SWF when there are exactly two alternatives. To prevent confusion of what is the majority rule SWF when generalized to multiple alter-
} 
Absolute Majority Rule SWF: Identical to the majority rule SWF, except it uses the strict balloting procedure.

Trivial Voting SWF: Identical to the majority rule SWF, except it uses the trivial balloting procedure.

Condorcet Method SWFs: Technically speaking, we are talking about a class of SWFs that share common features, as opposed to a single SWF. These SWFs' commonalities are as follows. First, the $n$ ! ballots allowed by the strict balloting procedure must be allowed by a Condorcet method SWF's balloting procedure. Now Condorcet method SWFs may allow more ballots than just those in the strict balloting procedure, but it must at least have those. Second with respect to the aggregation procedure, if there exists some alternative, say $a_{x}$, in the profile, such that $a_{x}$ defeats each of the other $n-1$ alternatives in the profile, one-on-one, via the majority aggregation procedure, then such an $a_{x}$ is ranked above all other alternatives in the social rank ordering of alternatives for that profile.

$U N V S W F$ : It uses the UNV balloting procedure and majority aggregation procedure.

For every logically possible pair $(A, V)$, given $\boldsymbol{A}$ and $\boldsymbol{V}$, a voting system assigns a social welfare function. If a voting system applies the same SWF to every possible $(A, V)$ given $\boldsymbol{A}$ and $\boldsymbol{V}$, then the voting system has the same name as the SWF. For example, the voting system which applies the UNV SWF to every possible $(A, V)$ given $\boldsymbol{A}$ and $\boldsymbol{V}$, is the UNV voting system. Note however, it is possible for a voting system to use different SWFs when $(A, V)$ changes. For example, we could define the crazy voting system as a voting system where, if $m$ is even it socially

natives, it helps to leave it undefined for $n>2$. Thus, technically speaking, the majority rule SWF would be what we later define as indecisive for $n>2$. But restricted to $n=2$, majority rule is decisive. Similarly for the absolute majority rule and trivial voting SWFs. 
rank orders alternatives in alphabetic order, and if $m$ is odd it socially rank orders alternatives in reverse alphabetic order.

\section{Four Arguments for Majority Rule}

Most defenses of Condorcet methods proceed from defending majority rule, and then asserting that if some alternative beats all other alternatives under consideration, one-on-one, via majority rule, then such an alternative should be the highest socially ranked.

Risse gives six reasons for majority rule (Risse 2004). Christian List gives three reasons (List 2013). While these are both excellent overviews of arguments for majority rule, in addition to the need for brevity, I will focus on the arguments put forth by Robert Dahl for two reasons. First, the arguments he gave largely overlapped with others', like Risse's and List's. Second, Dahl's arguments are probably among the most known and accepted arguments for majority rule in the political philosophy research community, so responding to them seems especially pertinent.

In his classic, Democracy and Its Critics, Dahl says that the four arguments he presents for majority rule are "... the strongest arguments for majority rule I know..." in the context of two alternatives (Dahl 1989, 136). After presenting his four arguments, Dahl discusses how problems arise when we allow more than two alternatives, and how there might be other decision procedures to consider, but how ultimately, while majority rule is imperfect, so are the other possible procedures (Dahl 1989, 135-162). We will proceed thru each of the four arguments, one by one, showing along the way how the UNV voting system does just as good or better of a normative job as Condorcet method voting systems and any other voting system at generalizing the argument to multiple alternatives.

May's Theorem: Majority Rule as a Necessary Consequence of Reasonable Requirements 
May's theorem uniquely characterized majority rule for two alternatives on an unrestricted balloting procedure with four normative conditions: decisiveness, anonymity, neutrality, and positive responsiveness. ${ }^{8}$ Dahl argues that the four conditions of May's theorem are conditions we'd want any reasonable voting system to have; this is roughly the argument Dahl makes for the four conditions (Dahl 1989, 139-141): We want decisiveness because we don't want situations where the voting system leaves the election undecided. ${ }^{9}$ We want anonymity and neutrality because we want voters to be treated equally, and (under certain boundary conditions, according to Dahl) we usually want alternatives to be treated equally. We want positive responsiveness, one, to prevent minority rule, and two, to ensure that if all voters but one is indifferent, the remaining voter's preference can determine the outcome in that remaining voter's favor. It just so happens, due to May's theorem, that when there are two alternatives, and voters are using the unrestricted balloting procedure, majority rule is the unique voting system that satisfies those four conditions.

Given Dahl's argument, one might be tempted to assert, that since majority rule is so normatively desirable, then any alternative that beats all other alternatives in $A$ one-on-one via majority rule should be socially ranked highest, regardless of the balloting procedure. This would superficially appear to be a natural generalization of May's theorem to multiple alternatives. But it's not quite so straightforward.

\footnotetext{
${ }^{8}$ Formal definitions of these four conditions are in the Supporting Information.

${ }^{9}$ Decisiveness can also be defended as an equality of expression condition, because roughly speaking, it says that if some voter is allowed to express some ballot, any other voter should be allowed to express that ballot.
} 
Goodin and List demonstrated that May's theorem was just a two-alternatives special case of a characterization of plurality voting system (or plurality rule as they called it) for multiple alternatives (Goodin and List 2006). Furthermore, it has since been shown that a very large number of voting systems, a voluminous number of which are not Condorcet methods, using a variety of SWF balloting procedures (including unrestricted, strict, and UNV balloting procedures) satisfy the four conditions (Prasad 2016). One of those voting systems is UNV. If UNV can satisfy the four conditions, there doesn't seem to be anything particularly advantageous for Condorcet methods with respect to May's theorem.

In addition to satisfying the four conditions, UNV satisfies those four conditions in a uniquely compelling way by essentially resolving what may be called May's dilemma. Specifically, May noted that with some restricted balloting procedure, one could satisfy his four conditions and Kenneth Arrow's independence of irrelevant alternatives (May 1952, 684). What he did not specify is which voting system, that satisfies the five conditions, uses SWFs with the least restrictive balloting procedure. As is clarified and proven in the May's dilemma (i.e. Theorem 1) portion of the Supporting Information, UNV is the unique voting system that uses SWFs with the least restrictive balloting procedure which satisfies decisiveness, anonymity, neutrality, positive responsiveness, and independence of irrelevant alternatives (IIA). Any other voting system that satisfies those five conditions must use SWF balloting procedures which are more restrictive than those used by the UNV system. The importance of these results is further clarified when we discuss the relationship between Arrow's theorem and May's theorem. $\underline{\text { Rae-Taylor Theorem: Maximization of Utility }}$

First off, we should make clear at the outset of this argument that with standard utility theory (i.e. von Neumann-Morgenstern utility theory), if at least one voter prefers $a_{x}$ over $a_{y}$ and 
at least one voter prefers $a_{y}$ over $a_{x}$, it is impossible to assert that $a_{x}$ or $a_{y}$ maximizes the overall utility of the $m$ voters. This is because in standard utility theory, interpersonal utilities are incomparable (K. J. Arrow 1963, 9-11). For example, if one voter assigns $x$ two utils and another voter assigns $x$ two utils, we cannot say with standard utility theory that both voters equally value $x$. Dahl was aware of this, but said in practice we are forced to make such nebulous utility assessments (Dahl 1989, 143). This leaves us two options. One, we could just say that arguments that voting systems maximize the overall utility of voters are practically false. Two, we could assume non-standard utility theories and make arguments on those bases. Given that Dahl took the second option, we will proceed in the same manner and assume non-standard utility theories.

Dahl's argument that majority rule maximizes utility is the Rae-Taylor theorem (List 2013; Dahl 1989, 142-144, 355-356). ${ }^{10}$ The theorem can be roughly stated as follows. Suppose that $m$ voters are confronted with a yes or no vote. Suppose that for any given voter, her prior probability of voting yes is $\rho$, and her prior probability of voting no is $1-\rho$, where $0<\rho<1$ and the $m$ voters' votes are identically and independently distributed (i.e. iid). After all voters vote, exactly one of two outcomes occur: yes wins or no wins. The question the Rae-Taylor theorem answers is which voting rule maximizes the probability that any given voter is in the winning coalition? ${ }^{11}$ If we assume that for any given voter, her utility from being a member of a winning

\footnotetext{
${ }^{10}$ Technically speaking, the Rae-Taylor theorem is a justification for what we would call abso-
} lute majority rule, not majority rule. That said, we will be able to generalize the Rae-Taylor theorem in a manner that makes this difference inconsequential.

11 Technically speaking, the Rae-Taylor theorem asks which voting rule minimizes the number of losing coalitions (i.e. maximizes the number of winning coalitions) any given voter is a member of over multiple elections. However, because the theorem assumes how voters vote from 
coalition is $u>0$ and her utility from being in a losing coalition is $-u$, then the Rae-Taylor theorem specifies which voting rules maximize the utility of any given voter. Informally, the question can be restated as follows: Suppose there are $m$ coins, one of which is gold while the remaining coins are silver. Each of the $m$ coins has a probability $\rho$ of landing heads, and the coin flips are all iid. Without your knowledge, each coin is flipped; if a coin lands heads, then it placed in an opaque "heads" urn; if a coin lands tails, then it is placed in an opaque "tails" urn. The only information you are given is the number of coins in each urn. Which urn should you choose to maximize your probability of choosing the urn with the gold coin?

In terms of our coin analogy, Douglas Rae and Michael Taylor showed you should always choose the urn with more coins. In other words, the Rae-Taylor theorem shows conditions under which absolute majority rule maximizes the probability of any given individual being in the winning coalition. ${ }^{12}$

What might be called the Rae-Taylor dilemma is: how is their theorem meaningfully generalized to multiple alternatives? What we prove in the Theorem 2 (i.e. Rae-Taylor dilemma) portion of the Supporting Information is that the Rae-Taylor theorem is a two-alternatives special case of a multiple-alternatives UNV theorem. Of course, there may also be a multiple alterna-

election to election is mutually independent, and because not being in the losing coalition is equivalent to being in the winning coalition, their question can be mathematically reduced to our statement of the question (Rae 1969; M. Taylor 1969).

${ }^{12}$ One could potentially normatively justify the equiprobability and statistical independence assumptions of the Rae-Taylor theorem via the cardinal utility and veil of ignorance arguments made in (Harsanyi 1953). 
tives generalization of the Rae-Taylor theorem using a voting system with a polychotomous ballot, but any such SWF will either fail one of May's conditions or IIA.

One potential criticism of using UNV to generalize the Rae-Taylor theorem is that it can appear to allow only two levels of utility; how can this be justified? Using a non-standard utility theory, one way to justify this is by making use of Herbert Simon's concept of satisficing, which has been well documented and studied in the psychology and economics literature (Simon 1955; Simon 1978; Simon 1987; Gigenrenzer, Todd and ABC Research Group 1999; Schwartz, et al. 2002; Byron 2004; Altman 2015). Specifically, each voter $i$ has her own aspiration level, which is a utility threshold, say $u_{i}(l)$, such that if the utility that $i$ receives from $a_{x}$, say $u_{i}(x)$, is greater than $u_{i}(l)$, then $i$ would consent to $a_{x}$. If $u_{i}(x)$ is less than or equal to $u_{i}(l)$, she would not consent to $a_{x}$.

When satisficing in practice, upon discovery of an alternative that is above their aspiration level, the individual chooses that alternative and terminates her search. With respect to satisficing in the context of UNV, a voter would place alternatives that she believes are above her aspiration level in the first part of her ballot, and the remaining alternatives in the second part. (If she believes all alternatives in $A$ satisfice, or that no alternative in $A$ satisfices, then she would submit the trivial ballot). Clearly, UNV in such a manner maximizes the number of voters who are satisficed; thus if we are using satisficing theory as our non-standard utility theory, then UNV can maximize utility.

\section{Maximization of Self-Determination}

In clarifying what he means by maximizing self-determination, Dahl states that "Given the boundaries of a particular political system, the composition of the demos, and the need for a collective decision on some matter... majority rule ensures that the greatest possible number of 
citizens will live under laws they have chosen for themselves" (Dahl 1989, 138). One way to interpret the meaning of "chosen for themselves" is focused on consent. One defense for a consent based notion of self-determination is that provided by satisficing utility theory, which we discussed earlier, because UNV maximizes consents. Another defense of self-determination as consent can be construed as contractual. As Dahl noted, we assume background conditions (i.e. political system, the composition of the demos, and need for a collective decision) as given (Dahl $1989,138)$. The question is what do we mean by choosing for oneself given fixed background conditions? It is impossible to give a full answer to that question here; that said, I wish to provide a brief sketch to show what an answer might look like.

For the sake of a concrete example, imagine there is a commons that can only be regulated in one way, but it is to be used by two different persons. We can imagine the two people having some sort of deliberative discourse, hammering out details, and developing a contract to which both would consent to, that would clarify the regulations on the commons.

Now suppose instead of two people, there were three people. In practice, this number of people is still small enough where we could imagine each person hammering out an individual contract with each of the other persons (i.e. person 1 with person 2, person 1 with person 3 , and person 2 with person 3), and each person making sure their own two contracts do not conflict with each other.

But if the number of people using the commons grows to four or five or $m$ persons, eventually, it will become unwieldy for each person to negotiate $m-1$ contracts that don't contradict each other, and the commons to have $(m-1)+(m-2)+\ldots+(1)=\left(m^{2}-m\right) / 2$ contracts which do not contradict each other. Eventually, there has to be another way. 
We could imagine one other way to be something like this. Suppose there are $m$ persons who must figure out how the commons will be regulated. We could imagine that there is some fair and reasonable deliberative discourse protocol. Eventually at some point, each individual is given the opportunity to propose potential contracts. We can call these potential contracts alternatives. Each of the $m$ persons, who are now voters, would mark each alternative to which they are willing to consent. If there exists some alternative which is consented by all voters, then such an alternative becomes the implemented contract.

Now this requirement for unanimous consent might work, especially if $m$ is small. But as $m$ becomes large, say a thousand or a million or a billion, there will be a point where the requirement for unanimous consent will become untenable because just one person is sufficient to prevent the implementation of some contract. Voting systems which use polychotomous SWFs, such as Condorcet methods, will either fail one of May's conditions or IIA.

However, in our commons example, especially if $m$ is large, UNV seems like a reasonable way to choose a contract because it maximizes consent while satisfying May's conditions and IIA. Furthermore, because consent is transitive, no supporters of losing alternatives can claim that their alternative lost to an alternative with less consent. Thus, UNV can be constructed such that it can maximize self-determination when voters are sincere.

$\underline{\text { Condorcet's Jury Theorem: More Likely to Produce Correct Decisions }}$

The simplest version of this argument goes something like this. Suppose there is some statement that is in exactly one of two states: true or false. (In this context, truth and falsity of the statement can be construed as two alternatives.) A group of $m$ voters is trying to determine whether the statement is true or false. Suppose that each voter in the group has the same probability $\pi$, where $0<\pi<1$, of correctly determining the state of the statement. Further assume that 
each voter's determination is mutually independent of the other voters' determinations. Condorcet's jury theorem shows that if $\pi>1 / 2$, then absolute majority rule more likely chooses the correct state than does absolute minority rule. Furthermore, under such conditions, as the number of voters increases, the probability that absolute majority rule chooses the correct state quickly, but asymptotically, approaches $100 \%$ accuracy.

In the 1980s, Peyton Young characterized a Condorcet method, often called the KemenyYoung method, which generalized the jury theorem to multiple alternatives (Young 1988). However, it is easy to show that the jury theorem can be generalized to UNV for multiple alternatives (Ben-Yashar and Kraus 2002).

In the simplest version, the generalization of the jury theorem to UNV can be stated as follows. Suppose there are $n$ statements, each of which is in exactly one of two states: true or false. There are $m$ voters. For any given statement, each voter has a probability $\pi$, where $0<\pi<$ 1 , of correctly determining the state of the given statement. Furthermore, each voter's determination for each statement is mutually independent of determinations on other statements and of determinations by other voters. If a given voter determines that a given statement is true, she places it in the first part of her ballot; if she determines it is false, she places it in the last part of her ballot. (Of course if a voter determines that all statements under consideration are true or that all are false, she will submit a trivial ballot). The generalization of the jury theorem to UNV asserts that if $\pi>1 / 2$, then UNV produces the rank ordering of statements from most likely true statement to least likely true statement (Prasad 2012). This result has been generalized in various ways for 
UNV (Brams and Kilgour 2014). ${ }^{13}$ Given that Condorcet's jury theorem can be generalized to multiple alternatives with UNV, the jury theorem does not seem to provide a notable normative reason as to why Condorcet methods would be superior to UNV.

But in addition to being able to construct a jury theorem result with UNV, it is also a unique voting system with respect to solving what may be called Condorcet's dilemma. Nicolas de Condorcet in the $18^{\text {th }}$ century had noticed a huge paradox with voting systems that tracked truth; specifically, it could be the case that a voting system chose the rank ordering of alternatives which was most likely true out of all possible rank orderings, but an alternative which such a rank ordering ranks above all other alternatives, has a lower probability of being true than some other alternative (Young 1988).

This problem can occur for at least two reasons. One, when there are polychotomous ballots, varying the $\pi$ value between $1 / 2$ and 1 can cause the problem (Young 1988, 1238). Two, because of the intransitivity of majority rule known as Condorcet's paradox. For example, under the conditions of Condorcet's jury theorem, whenever a majority prefers $a_{x}$ over $a_{y}$, then $a_{x}$ is more probably true than $a_{y}$ if they are the only two alternatives under consideration. If there are multiple alternatives, then it is possible that applying the majority aggregation procedure to each possible pair of alternatives could cause an intransitive cycle. But because SWFs are required to output a transitive preference order, an SWF may have to choose a rank ordering that goes against majority rule on some pairs of alternatives; thus, it may be forced to rank a less likely true alternative above alternatives which are more likely true (Young 1988).

\footnotetext{
${ }^{13}$ Technically, these generalizations are for approval voting, but the results easily follow for UNV when voters are truth seekers (i.e. prefer a true alternative over a false alternative, are indifferent between any true alternatives, and are indifferent between any false alternatives).
} 
After we informally clarify some conditions which are formally stated in the Theorem 3 (i.e. Condorcet's dilemma) portion of the Supporting Information, we can state our uniqueness result for UNV. First, the majority aggregation condition requires every SWF of the voting system use the majority aggregation procedure. (This first condition implies satisfaction of IIA.)

Second, roughly speaking, the epistemic voting system condition requires that there exist background conditions where the following two assertions are true about every SWF in a voting system that satisfies the condition: (1) under appropriate background conditions, a SWF outputs a rank ordering with $a_{x}>a_{y}$ iif $a_{x}$ is more probably true than $a_{y}$, and (2) under appropriate background conditions, a SWF outputs a rank ordering where $a_{x} \sim a_{y}$ iif $a_{x}$ and $a_{y}$ have an equal probability of being true. (In other words, our SWF is required to produces rank orderings which track truth under appropriate background conditions.)

With those normative conditions stated, we can roughly state Theorem 3 , which is proven in the Supporting Information: First, there exists no voting system, which regardless of background conditions, will satisfy the decisiveness, neutrality, majority aggregation, and epistemic voting system conditions. Second, regardless of background conditions, no polychotomous voting system can satisfy the decisiveness, neutrality, majority aggregation, and epistemic voting system conditions. Third, there exists a set of background conditions where UNV satisfies the decisiveness, neutrality, majority aggregation, and epistemic voting system conditions. Fourth and most importantly, UNV is the unique voting system with the least restrictive balloting procedure which has a set of background conditions which allow it to satisfy the decisiveness, neutrality, majority aggregation, and epistemic voting system conditions.

\section{Truth, Justice, and the General Will}


A major objection to UNV is the assertion that important political phenomena rarely occur in such a manner non-polychotomous preferences are sufficient for voters to express their opinion. I do not make the claim that every political phenomenon is sufficiently covered by two levels of preference. However, I do think they sufficiently cover many important political phenomena. I will discuss three kinds of phenomena. The hope is to demonstrate that UNV allows for sufficient expression in many different important political domains.

The first domain is truth, often associated with epistemic democratic theory. While there exist many-valued logics, two-valued logic is overwhelmingly the most used. If we seek our voting system to track some external objective truth (like the UNV generalization of the Condorcet jury theorem), UNV seems to be sufficient because alternatives are only in one of two states: true or not true. Assuming we are restricted to Arrovian voting systems, when alternatives are only in one of two states, is it necessary that voters be able to say something other than true or not true? Perhaps we could ask voters to assign probabilities of truth to each alternative, but this would be non-Arrovian. In addition to tracking objective truth, UNV can potentially also track truth by consensus. For example, each voter could be asked to choose each alternative which does not cause pain to her. If for the sake of argument, we assume pain is a subjective experience, then UNV can track the number of voters who do not feel pain from any given alternative, even though this is a truth that is based on the size of the number of voters in the consensus and not some external objective truth.

The second domain is Rawlsian justice. John Rawls believed that constitutions and legislation in a just society should be just. But what is the nature of this justice? For example, is it possible for two laws to be just, but one more just than the other? This is a complicated issue that 
merits more space, but there is evidence to suggest that Rawls believed that a large area of legislation and constitutions had to be guided by a binary notion of justice. Rawls states:

"Now the question of whether legislation is just or unjust, especially in connection with economic and social policies, is commonly subject to reasonable differences of opinion. In these cases judgment frequently depends upon speculative political and economic doctrines and upon social theory generally. Often the best that we can say of a law or policy is that it is at least not clearly unjust.... It is often perfectly plain and evident when the equal liberties [i.e. first principle] are violated.... But this state of affairs is comparatively rare with social and economic policies regulated by the difference principle" (Rawls 1999, 174).

“... [T] he four-stage sequence is a device for applying the principles of justice.... Of course, this test is often indeterminate: it is not always clear which of several constitutions, or economic and social arrangements, would be chosen. But when this is so, justice is to that extent likewise indeterminate. Institutions within the permitted range are equally just, meaning they could be chosen; they are compatible with all the constraints of the theory. Thus on many questions of social and economic policy we must fall back upon a notion of quasi-pure procedural justice: laws and policies are just provided that they lie within the allowed range...." (Rawls 1999, 176).

Our comments on binary truth and Rawlsian justice definitely deserve more in-depth analysis in another piece. But I hope they give some sense of the scope with which UNV allows for sufficient expression. The final example I wish to discuss is Jean-Jacques Rousseau's general will. Specifically, I want to show how UNV resolves a concern that is present with the general will when voters use polychotomous ballots.

Even in an ideal Rousseauian state, if voters are determining the general will through a series of majority rule elections, where ballots represent preference orders, then clearly the general will, "divined" from such elections, could be muddled with intransitive cycles due to the intransitivity of majority preference, even if no individual voter changes their opinions between elections.

UNV seems to better fit Rousseau's general will than methods using polychotomous ballots. Rousseau states: "When any law is proposed in the assembly of the people, the question is 
... if it is conformable or not to the general will (Rousseau 1947, 95)." Note, the voter is not asking himself if one proposed law is better than another with respect to the general will? The voter asks if a proposed law satisfies the general will or not? This is a binary question. If this is the case, UNV can ensure that the intransitive majority cycles possible with polychotomous ballots do not affect the general will.

\section{Responding to Riker's Criticism of Populist Democracy}

All of this is extremely crucial with respect to interpreting Rousseau and populist conceptions of democracy. William Riker, in his magnum opus, Liberalism Against Populism (1982), argued that populist conceptions of democracy, such as Rousseau's, were fatally flawed as they could not produce a meaningful aggregation of individual opinions into a general will, since all Arrovian voting systems are mired in the possibility of outputting intransitive cycles or violating some condition of Arrow's theorem (Riker 1982, 65-136). What our results here suggest is that individual opinions can potentially be meaningfully aggregated into some sort of general will.

Kenneth Arrow had two versions of his famous theorem, one from 1951 and one from 1963. ${ }^{14}$ The 1951 version used stronger conditions than the 1963 version,,${ }^{15}$ which means that had there existed a SWF which satisfied the conditions of the 1951 version, it would necessarily sat-

\footnotetext{
${ }^{14}$ Technically, Arrow's 1963 version is based on a French version he published in 1952 (Arrow
} 1963, 97).

${ }^{15}$ Technically speaking, Arrow's unrestricted domain condition from 1963 is stronger than his "condition 1" from 1951. But Arrow notes that this is only used to simplify exposition of the proof, and the proof of his 1963 version can still be done with something weaker than unrestricted domain (Arrow 1963, 97). 
isfy the conditions of the 1963 version. The fact that no SWF satisfies the conditions of the 1963 version implies that no SWF satisfies the conditions of the 1951 version (Arrow 1963).

The stronger versions of conditions are unrestricted domain, non-dictatorship, nonimposition, positive association, and Arrow's IIA. ${ }^{16}$ Note that non-dictatorship, non-imposition, positive association, and Arrow's IIA are weaker versions of anonymity, neutrality ${ }^{17}$, positive responsiveness, and IIA, respectively. ${ }^{18}$ Thus, if we could find a voting system which satisfied unrestricted domain, anonymity, neutrality, positive responsiveness, and IIA, it would satisfy the conditions of both versions of Arrow's theorem. Of course, Arrow's theorem shows no such voting system exists.

But suppose we were in a situation where we could legitimately assert that voters have (or at least should have) only non-polychotomous preferences. We might be able to assert this if we are dealing with particular situations where the decisions regard epistemic democratic truth or Rawlsian justice or Rousseauian general will in the manner we discussed earlier. If such is the case, then instead of having an unrestricted domain condition, it may be legitimate to have an

${ }^{16}$ These are the conditions from Arrow's 1951 version, with the exception of substituting Arrow's condition 1 from 1951 with the stronger unrestricted domain condition from 1963.

${ }^{17}$ Technically speaking, there exist SWFs which always output a tie between all alternatives and are neutral, but fail non-imposition. But such SWFs are useless. Among useful SWFs, that is among SWFs that do not always output a tie between all alternatives, any neutral useful SWF necessarily satisfies non-imposition.

${ }^{18}$ Note Arrow's IIA is defined for a fixed $A$ (Arrow 1963, 27). Our IIA allows the set of alternatives to vary. For example, the crazy voting system would satisfy Arrow's IIA but fail our version. Thus, our IIA is thus a stronger condition. 
unrestricted non-polychotomous domain condition, meaning we require the SWF to be decisive and for its balloting procedure to have all logically possible non-polychotomous ballots. But our characterization of UNV as uniquely satisfying May's conditions and IIA on the UNV procedure, which is a corollary of the approval voting theorem from (Goodin and List 2006), shows that if we replaced condition 1 (or unrestricted domain) with an unrestricted non-polychotomous domain condition, UNV could uniquely satisfy very strong versions of Arrow's theorem's remaining conditions. The claim being made here is not that the UNV balloting procedure is appropriate in every context; rather in contexts where the UNV balloting procedure is appropriate, Arrow's theorem can be practically overcome. Thus in those contexts, Riker's Arrow's theorem based criticism of populist democracy is not justified.

If democracy is epistemically (i.e. true or not true) or contractually (i.e. consent or not consent) based, it would suggest that Riker's Arrovian criticisms of populist democracy are significantly weaker. Future Rikerian criticism of populist democracy would have to rely more on his Gibbard-Satterthwaite theorem and strategic voting based arguments.

Overall, if one wishes to stay in the Arrovian framework and if one agrees with the arguments given by Dahl, our work here very strongly demonstrates one ought to generalize majority rule with UNV. The only other choices would seem to be to reject the Arrovian framework or the foundational arguments of majority rule.

\section{Supporting Information}

The reader is advised to read the Basic Definitions section prior to reading this section. This Supporting Information section is organized in the following manner. First, there is background which gives more definitions required for the proofs in this section. Second, Theorem 1 specifies the proof for the solution to May's dilemma. Third, Theorem 2 resolves the Rae-Taylor 
dilemma by providing the first generalization of the Rae-Taylor theorem to multiple alternatives, and shows how the Rae-Taylor theorem is a special case of this generalization when there are two alternatives. Fourth, Theorem 3 provides a solution to Condorcet's dilemma.

\section{Background}

The size of a part refers to the number of alternatives in the part. For example, for the ballot $\left(a_{w}\right)\left(a_{x} a_{y}\right)\left(a_{z}\right)$, the first part is of size 1 since it has only one alternative in it, the second is of size 2 , and the third of size 1 . In general, let us refer to the size of the first part of a ballot as $e_{1}$, the size of the second part as $e_{2}$, of the third part as $e_{3}$, and so forth. If two ballots on the same $A$ have the same number of alternatives, the same number of parts, and are such that (their $e_{1}$ is the same, their $e_{2}$ is the same, and so forth), then the two ballots are said to be of the same composition. For example, both $\left(a_{w}\right)\left(a_{x} a_{y}\right)\left(a_{z}\right)$ and $\left(a_{y}\right)\left(a_{w} a_{z}\right)\left(a_{x}\right)$ are of the same composition, but $\left(a_{w}\right)\left(a_{x}\right.$ $\left.a_{y}\right)\left(a_{z}\right)$ and $\left(a_{w} a_{x}\right)\left(a_{y}\right)\left(a_{z}\right)$ are not of the same composition.

The set of all ballots of a given composition is called a bundle. For example, if $A=\left\{a_{w}\right.$, $\left.a_{x}, a_{y}, a_{z}\right\}$, then the 1-2-1 composition bundle (i.e. the bundle of ballots where $e_{1}=1, e_{2}=2, e_{3}=$ 1) for $A$ would consist of the following twelve ballots: $\left(a_{w}\right)\left(a_{x} a_{y}\right)\left(a_{z}\right),\left(a_{w}\right)\left(a_{x} a_{z}\right)\left(a_{y}\right),\left(a_{w}\right)\left(a_{y}\right.$ $\left.a_{z}\right)\left(a_{x}\right),\left(a_{x}\right)\left(a_{w} a_{y}\right)\left(a_{z}\right),\left(a_{x}\right)\left(a_{w} a_{z}\right)\left(a_{y}\right),\left(a_{x}\right)\left(a_{y} a_{z}\right)\left(a_{w}\right),\left(a_{y}\right)\left(a_{w} a_{x}\right)\left(a_{z}\right),\left(a_{y}\right)\left(a_{w} a_{z}\right)\left(a_{x}\right),\left(a_{y}\right)\left(a_{x}\right.$ $\left.a_{z}\right)\left(a_{w}\right),\left(a_{z}\right)\left(a_{w} a_{x}\right)\left(a_{y}\right),\left(a_{z}\right)\left(a_{w} a_{y}\right)\left(a_{x}\right)$, and $\left(a_{z}\right)\left(a_{x} a_{y}\right)\left(a_{w}\right)$. That bundle is the list of all logically possible ballots of the 1-2-1 composition given $A=\left\{a_{w}, a_{x}, a_{y}, a_{z}\right\}$. In general, for a composition with $n$ alternatives and $\eta$ parts, where $e_{1}+e_{2}+\ldots+e_{\eta}=n$, the number of ballots in that composition bundle is $\left[n ! /\left(e_{1} ! e_{2} ! \ldots e_{\eta} !\right)\right]$.

If a given ballot occurs in a given balloting procedure, and every logically possible ballot with the same ballot composition as the given ballot also occurs in that given balloting proce- 
dure, then that given ballot is called a bundled ballot. If every ballot in a balloting procedure is a bundled ballot, then the balloting procedure is called a bundled balloting procedure.

For example, majority rule contains three bundled ballots and two bundles. If $A=\left\{a_{x}\right.$, $\left.a_{y}\right\}$, one bundle is the trivial composition ballot bundle, which has as its sole member, $\left(a_{x} a_{y}\right)$, and the other bundle is the 1-1 composition bundle which contains exactly two ballots, which are $\left(a_{x}\right)\left(a_{y}\right)$ and $\left(a_{y}\right)\left(a_{x}\right)$. In majority rule, since all ballots come bundled with all the other ballots of their respective bundle, all of majority rule's ballots are bundled ballots, and therefore its balloting procedure is a bundled balloting procedure.

May's theorem characterized majority rule with four normative conditions defined on two alternatives: decisiveness, anonymity, neutrality, and positive responsiveness. We will define those four conditions here but for SWFs with any $n$; our generalizations reduce to May's conditions when $n=2$.

Anonymity: A SWF is anonymous if for every profile in the SWF's profile domain, it is the case that if the ballots in a profile are permuted among voters to create a new profile (and this is the only change between the two profiles), then the output of the SWF is the same for both profiles. A voting system is anonymous if every SWF of the voting system is anonymous.

Decisiveness: A SWF is decisive if for each logically possible profile given the balloting procedure of the SWF, the SWF outputs a rank ordering. ${ }^{19}$ A voting system is decisive if every SWF of the voting system is decisive.

\footnotetext{
${ }^{19}$ In other words, if there are $c$ ballots in the balloting procedure of a SWF given a particular $A$ and particular $V$, then a decisive SWF is a total function which must output a rank ordering for each of the $c^{m}$ logically possible profiles.
} 
Neutrality: A SWF is neutral if for every profile in the SWF's profile domain, it is the case that a permutation of alternatives in a profile causes the alternatives to be permuted in the same manner in the outputted rank ordering. A voting system is neutral if every SWF of the voting system is neutral.

Positive Responsiveness: Positive responsiveness is the most complicated of May's four conditions to express. We will need to define a few terms to define positive responsiveness including raising $a_{x}$ relative to $a_{y}$, an $a_{x}$ positive flip relative to $a_{y}$, pre-flip profile, and post-flip profile. First, let $a_{x} \gtrsim a_{y}$ mean $a_{x}$ is ranked above or equal to $a_{y}$. Furthermore if we want to express the relation between two alternatives in a particular preference order, we will index it. For examples, if the ballot of voter $i$ expresses $a_{x}>a_{y}$, then this relation is expressed as $a_{x}>_{i} a_{y}$; if the rank ordering outputted for profile $p$ expresses $a_{x} \sim a_{y}$, then this relation is expressed as $a_{x} \sim p a_{y}$. Now suppose one of three things. One, a ballot has $a_{y}>a_{x}$ but changes to a ballot with $a_{x} \sim a_{y}$. Two, a ballot has $a_{y}>a_{x}$ but changes to a ballot with $a_{x}>a_{y}$. Three, a ballot has $a_{x} \sim a_{y}$, but changes to a ballot where $a_{x}>a_{y}$. (If either of these three things happen, we are not concerned with what happens with the other $\left(n^{2}-n-2\right) / 2$ pairwise comparisons on the ballot. As long as the ballot after the change is still a complete and transitive preference order, the other $\left(n^{2}-n-\right.$ 2)/2 pairwise comparisons in the ballot can change or remain the same.) If any of those three things occur, it is called raising $a_{x}$ relative to $a_{y}$.

Consider two profiles for which $A$ is the same for both profiles and $V$ is the same for both profiles. In each profile, there is exactly one voter $v_{j}$, and for every $v_{i}$, where $i \neq j$, it is the case that the ballot of $v_{i}$ is identical in both profiles. (This does not necessarily mean that the $m-1$ voters that are not $v_{j}$ have the same ballot in a given profile. All it is saying is that, for example, so long as $j \neq 1$ if $v_{1}$ has the ballot $\left(a_{x}\right)\left(a_{y} a_{z}\right)$ in one profile then $v_{1}$ has the ballot $\left(a_{x}\right)\left(a_{y} a_{z}\right)$ in the other 
profile, so long as $j \neq 2$ if $v_{2}$ has the ballot $\left(a_{y}\right)\left(a_{x}\right)\left(a_{z}\right)$ in one profile then $v_{2}$ has the ballot $\left(a_{y}\right)\left(a_{x}\right)\left(a_{z}\right)$ in the other profile, and so forth.) The only difference between the two profiles is the ballot submitted by $v_{j}$. Specifically, $v_{j}$ raises $a_{x}$ relative to $a_{y}$. The profile prior to the raising is called the pre-flip profile. The profile after the raising is called the post-flip profile. Such a change of a profile from a pre-flip to a post-flip profile is called an $a_{x}$ positive flip relative to $a_{y}$. In general, a SWF is positively responsive if it is the case for any $a_{x}$ and $a_{y}$ in $A$, that for every possible $a_{x}$ positive flip relative to $a_{y}$ (given the SWF's profile domain), where the SWF outputs $a_{x} \succsim a_{y}$ in the social rank ordering outputted for the pre-flip profile, the SWF also outputs $a_{x}>a_{y}$ in the social rank ordering outputted for the post-flip profile. A voting system is positively responsive if every SWF of the voting system is positively responsive.

Independence of Irrelevant Alternatives: Define $A_{2}$ as a set of two alternatives under consideration, namely the alternatives $a_{x}$ and $a_{y}$. Let $A_{+}$be a set of alternatives under consideration where $n$ $\geq 2$ and $A_{2}$ is a subset of $A_{+}$. Let $p_{2}$ be a profile on the set of alternatives $A_{2}$ and the set of voters $V$. Let $p_{+}$be a profile on the set of alternatives $A_{+}$and the set of voters $V$. Let $v_{i}^{2}\left(a_{x}, a_{y}\right)$ be defined as the pairwise comparison that the ballot of $v_{i}$ expresses between $a_{x}$ and $a_{y}$ in $p_{2}$, and let $v_{i}^{+}\left(a_{x}, a_{y}\right)$ be the pairwise comparison that the ballot of $v_{i}$ expresses between $a_{x}$ and $a_{y}$ in $p_{+}$. Assume that for every $i$, it is the case that $v_{i}^{2}\left(a_{x}, a_{y}\right)=v_{i}^{+}\left(a_{x}, a_{y}\right)$. If for every such $a_{x}$ and $a_{y}$ and every such $p_{2}$ and $p^{+}$, where both profiles are allowed by the voting system, the outputs of the voting system express the same pairwise comparison between $a_{x}$ and $a_{y}$ in its social rank orderings produced for both profiles, then such a voting system satisfies independence of irrelevant alternatives (IIA).

Approval voting is a non-Arrovian voting system, where as before, the set of alternatives is $A$ and the set of voters is $V$. Each voter marks each alternative to which she consents to. Alternatives 
are socially ranked ordered based on the number of marks they are given. (i.e. if $a_{x}$ is marked by more voters than $a_{y}$, then $a_{x}$ is socially ranked above $a_{y}$; if they receive the same number of marks, they are socially ranked equally.)

\section{Theorem 1: Solution to May's Dilemma}

Theorem 1: UNV is the unique Arrovian voting system that uses SWFs with the least restrictive balloting procedure that satisfy decisiveness, anonymity, neutrality, positive responsiveness, and IIA.

In other words, roughly speaking, any other Arrovian voting system that satisfies those five conditions must use SWFs with profile domains that are a proper subset of the UNV profile domain.

Proof Sketch: The proof consists of four stages. First, we identify all SWFs that satisfy decisiveness, anonymity, neutrality, and positive responsiveness for $n=2$. Those three SWFs are trivial voting, absolute majority rule, and majority rule, all of which use the majority aggregation procedure. Second, we demonstrate that any decisive voting system, where every one of its SWFs uses the majority aggregation procedure, must necessarily satisfy anonymity, positive responsiveness, and IIA. Third, we will show that any SWF balloting procedure that contains a polychotomous ballot will cause a voting system to fail either decisiveness, anonymity, neutrality, positive responsiveness, or IIA. This primarily entails demonstrating that SWFs that use polychotomous ballots will produce some intransitive rank ordering in order to satisfy the five conditions, which is a contradiction. Fourth, piggybacking off of work by Goodin and List, we can show that on the non-polychotomous balloting procedure, which is the balloting procedure that contains all logically possible ballots which are not polychotomous ballots, UNV uniquely satis- 
fies the five conditions. This fourth stage of the proof can be considered a corollary of GoodinList's approval voting theorem (Goodin and List 2006). ${ }^{20}$ Proof (Stage 1): Identifying all SWFs that Satisfy May's Four Conditions for $n=2$

First note, when alternatives are permuted within a ballot, the ballot composition will remain the same. This means that in order for a SWF to satisfy neutrality and decisiveness, it must use a bundled balloting procedure. When there are exactly two alternatives, there are exactly three possible bundled balloting procedures: trivial, strict, and unrestricted. In his theorem, May showed that majority rule uniquely satisfies decisiveness, anonymity, neutrality, and positive responsiveness on the unrestricted balloting procedure for $n=2$. It follows as corollaries that trivial voting (on the trivial balloting procedure) and absolute majority rule (on the strict balloting procedure) are the only other SWFs that satisfy May's four conditions on $n=2$.

Proof (Stage 2): Any Decisive Voting System which uses the Majority Aggregation Procedure (on Every Pair of Alternatives for Every One of Its SWFs) Must Necessarily Satisfy Anonymity, IIA, and Positive Responsiveness

First, for any pair of alternatives, $a_{x}$ and $a_{y}$, when ballots are permuted among voters, while it can change the profile (if the voting system is decisive), it does not change the number of voters who rank $a_{x}$ above $a_{y}$, nor does it change the number of voters who rank $a_{y}$ above $a_{x}$. Therefore the majority aggregation procedure would treat both profiles the same. Thus, every decisive voting system that uses the majority aggregation procedure on every pair of alternatives will be anonymous.

${ }^{20}$ The theorem can also be considered a corollary of (Inada 1964) or (Maniquet and Mongin 2015). 
Second, for any given pair of alternatives, $a_{x}$ and $a_{y}$, the majority aggregation procedure only uses voters' respective pairwise comparisons between $a_{x}$ and $a_{y}$. Therefore, any voting system where every one of its SWFs uses the majority aggregation procedure on every pair of alternatives will satisfy IIA.

Third, let us introduce some terminology. Let $n_{x}$ represent the number of voters who prefer $a_{x}$ over $a_{y}$ in a profile $p$, and let $n_{y}$ represent the number of voters who prefer $a_{y}$ over $a_{x}$ in the same profile $p$. For ease of expression, we say the numbers of voters in $p$ that prefer $a_{x}$ over $a_{y}$ and that prefer $a_{y}$ over $a_{x}$ is represented by $\left(n_{x}, n_{y}\right)$. Now suppose there is an $a_{x}$ positive flip relative to $a_{y}$ from $p$ to the profile $p^{\prime}$. Then it must be the case that $p^{\prime}$ is such that $\left(n_{x}+1, n_{y}\right),\left(n_{x}, n_{y}-1\right)$, or $\left(n_{x}+1, n_{y}-1\right)$. But if the majority aggregation procedure outputs a rank ordering for $p$ where $a_{x}$ $\gtrsim a_{y}$, then it must output a rank ordering for $p^{\prime}$ where $a_{x}>a_{y}$. Thus, a voting system which uses the majority aggregation procedure on every pair of alternatives must satisfy positive responsiveness.

Proof (Stage 3): Any Voting System with a SWF which uses a Polychotomous Ballot Must Fail One of May's Four Conditions or IIA

First note that there are exactly three SWFs which satisfy decisiveness, anonymity, neutrality, and positive responsiveness on exactly two alternatives: majority rule, absolute majority rule, and trivial voting. But each of those SWFs use the majority aggregation procedure. Therefore, any voting system that satisfies [(decisiveness, anonymity, neutrality, and positive responsiveness) on $n=2$ ] and IIA must be such that each of its SWFs uses the majority aggregation procedure on every pair of alternatives, regardless of $A$ or the value of $n$.

Now recall, if a decisive voting system is such that all of its SWFs use the majority aggregation procedure on every pair of alternatives, then the voting system necessarily satisfies an- 
onymity, positive responsiveness, and IIA. The question is if such a voting system can also be neutral and use a SWF which has a polychotomous ballot in its profile domain?

Consider any particular $A$ and $V$, say $A^{*}$ and $V^{*}$, where $n>2$. (Polychotomous ballots are impossible on $n=2$ ). Further consider a SWF, $f$. Suppose that the balloting procedure for $f$ contains some polychotomous ballot for an election with $A^{*}$ and $V^{*}$. Since $n>2$, there will at least be three alternatives; let us call three of those alternatives $a_{x}, a_{y}$, and $a_{z}$. Now note, every polychotomous ballot will have a first part, a second part, and a third part. Let " $\left(a_{x} \ldots\right)\left(a_{y} \ldots\right)\left(a_{z} \ldots\right) \ldots$ " refer to a particular ballot where $a_{x}$ occurs in the first part (where other alternatives might occur in the first part), $a_{y}$ occurs in the second part (where other alternatives might occur in the second part), $a_{z}$ occurs in the third part (where other alternatives might occur in the third part), and other alternatives exist in other parts of the ballot if the ballot has more than three parts. Let us say " $\left(a_{x} \ldots\right)\left(a_{y} \ldots\right)\left(a_{z} \ldots\right) \ldots$.. occurs in the balloting procedure of $f$ for $A^{*}$ and $V^{*}$. Note, given its description, such a ballot must exist in any SWF balloting procedure with a polychotomous ballot. If $f$ satisfies neutrality and decisiveness, then it must be the case that it uses a bundled balloting procedure; thus there must also exist particular ballots with the same composition as the " $\left(a_{x} \ldots\right)\left(a_{y} \ldots\right)\left(a_{z} \ldots\right) \ldots$ ". ballot that have the form “ $\left(a_{y} \ldots\right)\left(a_{z} \ldots\right)\left(a_{x} \ldots\right) \ldots$ " " $\left(a_{z} \ldots\right)\left(a_{x} \ldots\right)\left(a_{y} \ldots\right) \ldots$ ", and “ $\left(a_{z} \ldots\right)\left(a_{y} \ldots\right)\left(a_{x} \ldots\right) \ldots$... To simplify notation for these particular ballots, let us call " $\left(a_{x} \ldots\right)\left(a_{y} \ldots\right)\left(a_{z} \ldots\right) \ldots$ ". the $x$-ballot, " $\left(a_{y} \ldots\right)\left(a_{z} \ldots\right)\left(a_{x} \ldots\right) \ldots$ " the $y$-ballot, " $\left(a_{z} \ldots\right)\left(a_{x} \ldots\right)\left(a_{y} \ldots\right) \ldots "$ the $z$-ballot, and " $\left(a_{z} \ldots\right)\left(a_{y} \ldots\right)\left(a_{x} \ldots\right) \ldots$ ". the anti-x-ballot.

Now given there are $m$ voters, let $\lfloor m / 3\rfloor$ be the floor function of $m / 3$. We will now proceed through all possible cases and show there exists some profile that would require a decisive, anonymous, neutral, positively responsive, and IIA satisfying voting system have a SWF which is intransitive, which contradicts the definition of a SWF. 
Case: $m=2$

Exactly one voter submits the $x$-ballot and exactly one voter submits the $z$-ballot. Note by majority aggregation procedure, $a_{x}>_{p} a_{y}$ and $a_{y} \sim p a_{z}$. Thus, by transitivity, $a_{x}>_{p} a_{z}$, but by majority aggregation procedure, $a_{x} \sim p a_{z}$. Thus, we have a contradiction. In theory, we can generalize this case to profiles where $m$ is even and $n>2$. However we will still discuss other cases where $m$ is even in order to produce examples where the cycle is caused without social ties. Cases: $m=3, m=6, m>8$

Consider a profile where $\lfloor m / 3\rfloor$ voters submit the $x$-ballot, another $\lfloor m / 3\rfloor$ voters submit the $y$-ballot, and the remaining $\lfloor m / 3\rfloor$ voters submit the $z$-ballot. Application of the majority aggregation procedure to each possible pair of alternatives in the profile will cause an intransitive cycle where $a_{x}>_{p} a_{y}, a_{y}>_{p} a_{z}$, and $a_{z}>_{p} a_{x}$. (If $m>8$, this occurs regardless of what the remaining (zero, one, or two) ballots in the profile are.)

Cases: $m=4, m=7$

Consider a profile where $\lfloor m / 3\rfloor+1$ voters submit the $x$-ballot, another $\lfloor m / 3\rfloor$ voters submit the $y$-ballot, and the remaining $[m / 3\rfloor$ voters submit the $z$-ballot. This profile has a majority cycle where $a_{x}>_{p} a_{y}, a_{y}>_{p} a_{z}$, and $a_{x} \sim p a_{z}$.

Cases: $m=5, m=8$

Consider a profile where $\lfloor m / 3\rfloor+1$ voters submit the $x$-ballot, another $\lfloor m / 3\rfloor$ voters submit the $y$-ballot, yet another $\lfloor m / 3\rfloor$ voters submit the $z$-ballot, and one voter submits the anti$x$-ballot. This profile has a majority cycle where $a_{x}>_{p} a_{y}, a_{y}>_{p} a_{z}$, and $a_{z}>_{p} a_{x}$.

\section{Proof (Stage 4): Uniquely Characterizing UNV}

The non-polychotomous balloting procedure is the least restrictive Arrovian balloting procedure (and least restrictive profile domain) without a polychotomous ballot, since it includes 
all non-polychotomous ballots and is decisive. Goodin and List effectively proved that UNV is unique on the non-polychotomous balloting procedure in terms of satisfying the five conditions, but for approval voting (Goodin and List 2006). Here, we give a proof using our terminology that UNV uniquely satisfies the five conditions on the non-polychotomous balloting procedure/profile domain.

Note, as demonstrated earlier, that the only three SWFs which satisfy May's four conditions when $n=2$ are majority rule, absolute majority rule, and trivial voting. All three use the majority aggregation procedure. Therefore, if there exists a voting system which satisfies May's four conditions and IIA on the non-polychotomous profile domain, it will be equivalent to a voting system, where all of its SWFs apply the majority aggregation procedure to each of their respective $\left(n^{2}-n\right) / 2$ pairs of alternatives. That unique SWF is UNV. And it is easy to verify that the UNV system is decisive, anonymous, neutral, positively responsive, and IIA.

\section{Theorem 2: A Solution to the Rae-Taylor Dilemma}

As usual, assume there are $n$ alternatives and $m$ voters. As with the original Rae-Taylor theorem, for any given alternative, $a_{x}$, any given voter has exactly one of two possible opinions, yes or no; we will assume that each voter has exactly two levels of utility on an alternative. For simplicity, we will say that for any voter $v_{i}$, the utility she gets from the implementation of an alternative, say $a_{x}$, to which she holds the opinion yes is $u_{i}(x)=u$, where $u>0$; the utility she gets from an alternative to which she holds the opinion no is $-u$.

Now imagine a $2^{n}$ sided die. ${ }^{21}$ Each side states an $n$-digit binary number, such that all binary numbers of $n$ length are expressed on the die. (For example, if $n=3$, then each of the fol-

${ }^{21}$ The die throwing discussed here is only used as a representation of the independent trials occurring when voters vote. Die throwing is the classical illustration of the multinomial distribu- 
lowing binary numbers will occur on the eight-sided die: 000, 001, 010, 011, 100, 101, 110, and 111.) Exactly one binary number is expressed on each side of the die. Thus in general, let $d_{000 \ldots 000,} d_{000 \ldots 001}, \ldots, d_{111 \ldots 111}$ represent each of the $2^{n}$ sides of the die. Let $0 \leq \rho\left(d_{000 \ldots 000}\right) \leq 1$ be the probability that the side $d_{000 \ldots . .000}$ is thrown. Similarly define the probability of any other side being thrown. By definition, $\rho\left(d_{000 \ldots 000}\right)+\rho\left(d_{000 \ldots 001}\right)+\ldots \rho\left(d_{111 \ldots 111}\right)=1$.

Each of the $m$ voters rolls the die once. However, no voter knows the result of any of the $m$ die rolls. Instead for each roll, a recorder, who is not a voter, records which voter rolled the die and what side the voter had thrown. The side thrown by a voter represents which alternatives she holds the opinion yes for, and which alternatives she holds the opinion no for. In general, a one in the $x$ th digit of the side thrown means the voter who threw that die holds yes for $a_{x}$, while a zero for that digit would have meant she holds no for $a_{x}$. (For example, if a voter throws the side $d_{00101}$, this means that for alternatives $a_{1}, a_{2}$, and $a_{4}$ she holds no, but for alternatives $a_{3}$ and $a_{5}$ she holds yes.)

After the $m$ die rolls, the recorder constructs a list which specifies the number of yeses each alternative got (which of courses implies the number of noes each alternative got). Let $k_{x}$ be the number of yeses $a_{x}$ received. As such, a list will take the form $\left(k_{1}, k_{2}, \ldots, k_{n}\right)$. The recorder chooses one voter. The recorder gives the chosen voter the list. The chosen voter must then select exactly one alternative. After the chosen voter has selected an alternative, the recorder will inform the chosen voter whether she threw yes or no for the selected alternative. If she had thrown yes, she receives $u$ utils, and if she had thrown no, she receives $-u$ utils. Which alternative should she choose to maximize her expected utility?

tion, which is the underlying distribution for iid die throws. This simplifies exposition and comprehension of the underlying math. 
Let us be clear about what knowledge the chosen voter has. She knows the values of $\rho\left(d_{000 \ldots} \ldots 00\right), \rho\left(d_{000 \ldots 001}\right), \ldots$, and $\rho\left(d_{111 \ldots 111}\right)$. She knows that the $m$ dies rolls are identically and independently distributed. She is not given information on who threw what side, or how many voters threw any given side. The only information she has about the rolls is the information provided in the recorder's list.

First, for any given alternative $a_{x}$, if $k_{x}$ voters said yes on $a_{x}$, then the probability that the chosen voter said yes on $a_{x}$ is $k_{x} / m$. This is because the die rolls were identically and independently distributed. Therefore, if she selects $a_{x}$ her expected utility is $\left[u\left(k_{x} / m\right)\right]+\left[(-u)\left(m-k_{x}\right) / m\right]$ $=(u)\left(2 k_{x}-m\right) / m$. When deciding between any two alternatives, $a_{x}$ and $a_{y}$, the chosen voter should choose $a_{x}$ when $(u)\left(2 k_{x}-m\right) / m>(u)\left(2 k_{y}-m\right) / m$. But $(u)\left(2 k_{x}-m\right) / m>(u)\left(2 k_{y}-m\right) / m$ iff $k_{x}>k_{y}$. Furthermore, $(u)\left(2 k_{x}-m\right) / m=(u)\left(2 k_{y}-m\right) / m$ iff $k_{x}=k_{y}$.

From this it is easy to see that the voter should select the alternative which has the most yeses in order to maximize her utility. If two or more alternatives are tied for most yeses, then the chosen voter can select any of the alternatives tied for most yeses. Thus, approval voting is a voting system which will maximize her utility.

Now technically, approval voting is a non-Arrovian voting system. That said, UNV can produce identical results as approval voting under the following assumptions. First, if a voter throws all zeros or all ones, then the recorder can document it as a trivial ballot, because the voter holds the same utility for all alternatives. If a voter throws at least one zero and at least one one, the recorder can document it as a dichotomous ballot, where alternatives that got ones are recorded in the first part of the ballot, and alternatives that got zeros are recorded in the second part of the ballot. (This is the recorder simply transcribing utilities as preference orders.) When creating the list to provide to the chosen voter, the list is instead $\left(\kappa_{1}, \kappa_{2}, \ldots, \kappa_{n}\right)$, where $\kappa_{x}$ is the 
number of ballots where $a_{x}$ occurs in the first part. As such, UNV will track approval voting, and thus maximize the utility of the chosen voter.

The Rae-Taylor theorem is a special case of this more general result. To see how, suppose $n=2$, $\rho\left(d_{00}\right)=\rho\left(d_{11}\right)=0,0<\rho\left(d_{01}\right)<1$, and $\rho\left(d_{10}\right)=1-\rho\left(d_{01}\right)$. These are the conditions of the RaeTaylor theorem. Our generalized theorem states that under these conditions, the chosen voter should select $a_{1}$ when $\kappa_{1}>\kappa_{2}, a_{2}$ when $\kappa_{1}<\kappa_{2}$, and either when $\kappa_{1}=\kappa_{2}$. But this is exactly what the Rae-Taylor theorem specifies.

\section{Theorem 3: A Solution to Condorcet's Dilemma}

$\underline{\text { Outline }}$

First we will provide some definitions to simplify exposition. This is followed by a statement of Theorem 3. The proof consists of five lemmas and the elaboration of three theorems. Informally speaking: The first lemma demonstrates that for every voting system, there exists a set of background conditions, where the voting system will fail at least one of the four normative conditions (i.e. decisiveness, neutrality, majority aggregation, and epistemic conditions); thus no voting system always satisfies the four normative conditions. The second lemma demonstrates that regardless of background conditions, every polychotomous voting system will fail at least one of the four normative conditions; thus no polychotomous voting system can ever satisfy all four normative conditions. The third, fourth, and fifth lemmas are used to demonstrate a conditional claim: If there exists a set of background conditions where the UNV voting system satisfies the four normative conditions, then the UNV voting system is the least restrictive voting system which possibly satisfies the four normative conditions. Finally, elaborating on two existing theorems (which we call the Condorcet Jury Theorem and Generalized Condorcet Jury Theorem), we easily demonstrate thru a corollary (which we call the Generalized Arrovian Condorcet 
Jury Theorem), that there exist background conditions under which the UNV voting system satisfies the four normative conditions. Thus, UNV is the least restrictive voting system which can possibly satisfy the four normative conditions. After the proof is completed, there is an informal discussion of a possible normative objection to Theorem 3.

\section{$\underline{\text { Definitions }}$}

A polychotomous profile is a profile which contains a polychotomous ballot. A polychotomous profile domain is a profile domain which contains a polychotomous profile. A polychotomous SWF is a SWF which has a polychotomous profile domain. A polychotomous voting system is a voting system which has a polychotomous SWF.

A non-polychotomous profile is a profile which contains no polychotomous ballot. A non-polychotomous profile domain is a profile domain which contains no polychotomous profile. A non-polychotomous $S W F$ is a SWF which does not have a polychotomous profile domain. A non-polychotomous voting system is a voting system which has no polychotomous SWF.

The $U N V$ profile domain (for a given $(A, V)$ ) contains no polychotomous profiles but contains every non-polychotomous profile (possible given $(A, V)$ ).

\section{The Four Normative Conditions}

The four normative conditions are decisiveness, neutrality, epistemic, and majority aggregation conditions. The first two have been defined previously. We define the latter two here. Epistemic Voting System (epistemic): A voting system satisfies the epistemic condition if every one of its SWFs satisfies the epistemic condition. A SWF $f$ satisfies the epistemic condition if there exists a set of background conditions where: (For every $a_{x}$ and $a_{y}$ in $A$ and every $p$ in the profile domain of $f$, it is the case that $f(p)$ has $a_{x}>_{p} a_{y}$ iff the likelihood of $a_{x}$ in fact being true is greater than the likelihood of $a_{y}$ in fact being true. For every $a_{x}$ and $a_{y}$ in $A$ and every $p$ in the 
profile domain of $f$, it is the case that $f(p)$ has $a_{y}>_{p} a_{x}$ iff the likelihood of $a_{y}$ in fact being true is greater than the likelihood of $a_{x}$ in fact being true. For every $a_{x}$ and $a_{y}$ in $A$ and every $p$ in the profile domain of $f$, it is the case that $f(p)$ has $a_{x} \sim p a_{y}$ iff the likelihood of $a_{x}$ in fact being true is equal to the likelihood of $a_{y}$ in fact being true.)

Majority Aggregation Condition: A SWF satisfies the majority aggregation condition iff it uses the majority aggregation procedure. A voting system satisfies the majority aggregation condition iff every SWF of that voting system uses the majority aggregation procedure.

\section{Least Restrictive}

Given a fixed $(A, V)$, Let $f$ be any SWF which satisfies the decisiveness, neutrality, majority aggregation, and epistemic normative conditions. Let $f^{\prime}$ be a particular such $f$, and $f^{*}$ be any $f\left(\right.$ where $f^{*} \neq f^{\prime}$ ). If it is the case for every $f^{*}$ that the profile domain of $f^{*}$ is a proper subset of the profile domain of $f^{\prime}$, then $f^{\prime}$ is the least restrictive $S W F$ which satisfies the four normative conditions. If a voting system uses the least restrictive SWF on every possible $(A, V)$ given $(A, V)$, then such a voting system is the least restrictive voting system.

\section{$\underline{\text { Statement of Theorem } 3}$}

Under appropriate background conditions, every voting system will fail at least one of the four normative conditions (i.e. decisiveness, neutrality, majority aggregation, and epistemic). Regardless of background conditions, every polychotomous voting system will always fail at least one of the four normative conditions. There exists some set of background conditions, where UNV satisfies the four normative conditions. UNV is the unique least restrictive voting system which can possibly satisfy the four normative conditions.

\section{Statement of Lemma 3.1}

No voting system always satisfies all four normative conditions. 


\section{Proof of Lemma 3.1}

Assume each alternative is in exactly one of two states: true or not true. Assume each voter has a probability $\pi=0$ of correctly determining the state of any given alternative. Assume a voter prefers an alternative she believes is true over any alternative she believes is not true. Assume a voter always honestly expresses her preferences on her ballot. Under such background conditions, every voting system will either fail the epistemic or majority aggregation conditions.

\section{Discussion of Lemma 3.1}

Given there is no voting system which can always satisfy all four normative conditions, our search will turn to identifying voting systems which can satisfy the four normative conditions (under appropriate background conditions), and to those that can never satisfy the four normative conditions (regardless of the background conditions). In this vein, we first turn our attention to polychotomous voting systems.

\section{Statement of Lemma 3.2}

Regardless of the background conditions, every polychotomous voting system will fail at least one of the four normative conditions.

\section{$\underline{\text { Proof of Lemma } 3.2}$}

The epistemic voting system and majority aggregation conditions require that every SWF, used by the voting system, use the majority aggregation procedure on every profile in the profile domain. But Theorem 1 showed there exists no polychotomous voting system, which is decisive and neutral and that always uses the majority aggregation procedure. Thus, a polychotomous voting system is always doomed to fail at least one of the four normative conditions.

\section{Discussion of Lemma 3.2}


Given no polychotomous voting system can ever satisfy all four conditions, regardless of the background conditions, we turn our attention to non-polychotomous voting systems.

\section{Statement of Lemma 3.3}

Every logically possible non-polychotomous profile domain (given $(A, V)$ ) is a subset of the profiles belonging to the UNV profile domain (on that given $(A, V)$ ). (Proof follows from the definition of the UNV profile domain.)

\section{$\underline{\text { Statement of Lemma } 3.4}$}

For each profile domain, there is exactly one SWF which uses the majority aggregation procedure. Thus, if a profile domain belongs to a SWF (which belongs to a voting system, which satisfies the four normative conditions given a particular set of background conditions), that SWF is the unique SWF which satisfies those four normative conditions on that profile domain (given that particular set of background conditions).

\section{Proof of Lemma 3.4}

Roughly speaking, a SWF is nothing more than a profile domain and aggregation procedure. Once a profile domain is specified and the aggregation procedure is specified, that SWF is specified also. Note, the majority aggregation condition on voting systems requires every SWF of the voting system to use the majority aggregation procedure. Thus, if a profile domain is used by an SWF, which is used by a voting system, which satisfies the four normative conditions (on an appropriate set of background conditions), that SWF must be the unique SWF on that profile domain which uses the majority aggregation procedure.

\section{Statement of Lemma 3.5}


If the UNV system satisfies the four normative conditions (given a particular set of background conditions), then UNV is the least restrictive voting system which can possibly satisfy the four normative conditions.

\section{Proof of Lemma 3.5}

First note, by definition, every SWF of the UNV voting system uses the UNV profile domain, and the majority aggregation procedure. Because polychotomous voting systems will always fail at least one of the four normative conditions (Lemma 3.2), it follows from Lemmas 3.3 and 3.4 that if UNV system satisfies the four normative definitions on a particular set of background conditions, then it is the least restrictive voting system which can do so.

\section{Discussion of Lemma 3.5 \& the Setup for the Rest of the Proof}

What remains to be demonstrated is that UNV, under particular background conditions, satisfies the four conditions. That proof is done in three steps. First, we state the original Condorcet jury theorem to demonstrate in fact, that we are generalizing this original version. Second, we generalize the Condorcet jury theorem to multiple alternatives using an existing generalization that uses approval voting. We call this the Generalized Condorcet Jury Theorem. Third, because approval voting is technically non-Arrovian, we translate the generalization with approval voting into a generalization with UNV. We call this generalization the Generalized Arrovian Condorcet Jury Theorem. Because the background conditions for the Generalized Arrovian Condorcet Jury Theorem are background conditions under which the four normative conditions are satisfied, the UNV system is the least restrictive voting system which can possibly satisfy all four normative conditions.

\section{Condorcet Jury Theorem}

\section{Condorcet Jury Theorem Background Conditions}


Suppose there is exactly one alternative, $a_{x}$, which is in exactly one of two states: true or not true. Suppose there are $m$ voters. Each voter has the same probability, $\pi$, where $1 / 2<\pi<1$, of correctly determining the state of $a_{x}$. Each voter's determination is mutually independent of the other $m-1$ determinations. When a voter votes, she honestly states her determination on $a_{x}$. Statement of Condorcet's Jury Theorem

If the Condorcet Jury Theorem Background Conditions are fulfilled, then the following is true: If more voters believe $a_{x}$ is true than believe it is not true, then it is more likely the case that $a_{x}$ is true in fact than that $a_{x}$ is not true in fact. If more voters believe $a_{x}$ is not true than believe it is true, then it is more likely the case that $a_{x}$ is not true in fact than that $a_{x}$ is true in fact. If an equal number of voters believe $a_{x}$ is true as believe it is not true, then the likelihood that it is in fact the case that $a_{x}$ is true is equal to the likelihood of the case that $a_{x}$ is in fact not true.

\section{Generalized Condorcet Jury Theorem}

\section{Generalized Condorcet Jury Theorem Background Conditions}

Suppose there are $n$ alternatives, each of which is in exactly one of two states: true or not true. Suppose there are $m$ voters. Each voter has the same probability, $1 / 2<\pi<1$, of correctly determining the state of any given alternative. ${ }^{22}$ For any voter, say $v_{i}$, and for any alternative, say $a_{x}$, the determination $v_{i}$ makes on $a_{x}$ is mutually independent of the other $m n-1$ determinations. When a voter votes, every voter on every alternative honestly states her determination.

\section{Statement of the Generalized Condorcet Jury Theorem}

If the Generalized Condorcet Jury Theorem Background Conditions are fulfilled, then the following is true: For any $a_{x}$ and any $a_{y}$, if more voters believe $a_{x}$ is true than believe $a_{y}$ is true, then it is more likely the case that $a_{x}$ is in fact true than that $a_{y}$ is in fact true. For any $a_{x}$ and any

\footnotetext{
${ }^{22}$ In other words, there are $m$ voters, each making $n$ determinations. Let $\pi_{i x}$ be the probability that voter $i$ correctly determines the state of alternative $x$, and similarly $\pi_{j y}$ for voter $j$ on alternative $y$. For any $v_{i}$ and $v_{j}$, and for any $a_{x}$ and $a_{y}, \pi_{i x}=\pi_{j y}=\pi$, where $1 / 2<\pi<1$.
} 
$a_{y}$, if more voters believe $a_{y}$ is true than believe $a_{x}$ is true, then it is more likely the case that $a_{y}$ is in fact true than that $a_{x}$ is in fact true. If an equal number of voters believe $a_{x}$ is true as believe $a_{y}$ is true, then the likelihood that it is in fact the case that $a_{x}$ is true is equal to the likelihood of the case that $a_{y}$ is in fact true.

Proof of the Generalized Condorcet Jury Theorem

Given the background conditions of the Condorcet Jury Theorem or the Generalized Condorcet Jury Theorem, suppose $k$ voters, where $0 \leq k \leq m$, believe $a_{x}$ is true. If such is the case, then we know that the likelihood that $a_{x}$ is in fact true is equal to:

Eq. 1: $\left(\begin{array}{c}m \\ k\end{array}\right)\left[\pi^{k}(1-\pi)^{m-k}\right] /\left(\left[\left(\begin{array}{c}m \\ k\end{array}\right)\left[\pi^{k}(1-\pi)^{m-k}\right]+\left(\begin{array}{c}m \\ k\end{array}\right)\left[\pi^{m-k}(1-\pi)^{k}\right]\right)\right.$

Therefore, given the Generalized Condorcet Jury Theorem Background Conditions, where $k$ voters believe $a_{x}$ is true, and $l$ voters, where $0 \leq l<k$, believe $a_{y}$ is true, $a_{x}$ has a greater likelihood of being in fact true than $a_{y}$ if:

Eq. 2: $\left(\begin{array}{c}m \\ k\end{array}\right)\left[\pi^{k}(1-\pi)^{m-k}\right] /\left(\left[\left(\begin{array}{c}m \\ k\end{array}\right)\left[\pi^{k}(1-\pi)^{m-k}\right]+\left(\begin{array}{c}m \\ k\end{array}\right)\left[\pi^{m-k}(1-\pi)^{k}\right]\right)>\right.$

$$
\left(\begin{array}{c}
m \\
l
\end{array}\right)\left[\pi^{l}(1-\pi)^{m-l}\right] /\left(\left[\left(\begin{array}{c}
m \\
l
\end{array}\right)\left[\pi^{l}(1-\pi)^{m-l}\right]+\left(\begin{array}{c}
m \\
l
\end{array}\right)\left[\pi^{m-l}(1-\pi)^{l}\right]\right)\right.
$$

Using algebra to solve Eq. 2, we find that Eq. 2 is only true if $\pi>1 / 2$. Thus, we can demonstrate that if more voters believe $a_{x}$ is true than believe $a_{y}$ is true, $a_{x}$ is more likely in fact true than $a_{y}$ given the background conditions of the Generalized Condorcet Jury Theorem. Similarly, we can show that if an equal number of voters believes $a_{x}$ is true as believe $a_{y}$ is true, then $a_{x}$ and $a_{y}$ have the same likelihood of in fact being true, given the background conditions of the Generalized Condorcet Jury Theorem.

Generalized Arrovian Condorcet Jury Theorem Generalized Arrovian Condorcet Jury Theorem Background Conditions 
Suppose there are $n$ alternatives, each of which is in exactly one of two states: true or not true. Suppose there are $m$ voters. Each voter has the same probability, $1 / 2<\pi<1$, of correctly determining the state of any given alternative. ${ }^{23}$ For any voter, say $v_{i}$, and for any alternative, say $a_{x}$, the determination $v_{i}$ makes on $a_{x}$ is mutually independent of the other $m n-1$ determinations. For any $v_{i}$ and for any $a_{x}$ and $a_{y}$, the following are true: If $v_{i}$ believes both $a_{x}$ and $a_{y}$ are true or that both are not true, then $a_{x} \sim_{i} a_{y}$. If $v_{i}$ believes $a_{x}$ is true but $a_{y}$ is not true, then $a_{x}>_{i} a_{y}$. When a voter votes, every voters submits a ballot which honestly reflects her preferences. Thus, given the background conditions of the Generalized Arrovian Condorcet Jury Theorem, each voter will submit some non-polychotomous ballot.

\section{Statement of the Generalized Arrovian Condorcet Jury Theorem}

If the Generalized Arrovian Condorcet Jury Theorem Background Conditions are fulfilled, then the following is true: For any $a_{x}$ and any $a_{y}$ in $A$, if UNV has $a_{x}>_{p} a_{y}$, then it is more likely the case that $a_{x}$ is in fact true than that $a_{y}$ is in fact true. For any $a_{x}$ and any $a_{y}$ in $A$, if UNV has $a_{y}>_{p} a_{x}$, then it is more likely the case that $a_{y}$ is in fact true than that $a_{x}$ is in fact true. For any $a_{x}$ and any $a_{y}$ in $A$, if UNV has $a_{x} \sim p a_{y}$, then the likelihood that it is the case that $a_{x}$ is in fact true is equal to the likelihood of the case that $a_{y}$ is in fact true.

\section{Proof of the Generalized Arrovian Condorcet Jury Theorem}

The proof of the Generalized Arrovian Condorcet Jury Theorem easily follows from the proof of the Generalized Condorcet Jury Theorem. Thus, there exist background conditions under which UNV satisfies the four normative conditions, and due to Lemma 3.5 UNV is the unique least restrictive voting system to satisfy the four normative conditions.

\section{Discussion}

\footnotetext{
${ }^{23}$ In other words, there are $m$ voters, each making $n$ determinations. Let $\pi_{i x}$ be the probability that voter $i$ correctly determines the state of alternative $x$, and similarly $\pi_{j y}$ for voter $j$ on alternative $y$. For any $v_{i}$ and $v_{j}$, and for any $a_{x}$ and $a_{y}, \pi_{i x}=\pi_{j y}=\pi$, where $1 / 2<\pi<1$.
} 
We will informally discuss one potential objection to Theorem 3. Suppose the assertion is made that even if a polychotomous voting system fails at least one of the four normative conditions, this may not matter because of the way that voters vote, normative condition violations never occur in practice. For example, suppose $\pi=1$ for all $m n$ determinations, voters prefer true alternatives over not-true alternatives, and sincerely express their preferences on their ballots; further suppose a polychotomous voting system uses the unrestricted profile domain and majority aggregation procedure for every SWF. Clearly, such a voting system will technically be indecisive for any profile which leads to an intransitive majority cycle, but given voters will never vote in a way to produce such a cycle, it does not practically matter.

The reply to this kind of objection would be to introduce a non-zero probability condition, which requires that every profile in the profile domain have a non-zero probability of occurring. As such, trouble causing profiles will have to occur, which means normative condition violations are still practically possible. Even if the probability of such normative condition violations are near zero, if the decision being made is important enough, the non-zero probability of failure will still matter. It would take a super idealized society of voters to overcome the nonzero probability condition.

\section{References}

Altman, Morris. 2015. "Satisficing." In Real-World Decision Making: An Encyclopedia of Behavioral Economics, edited by Morris Altman, 374-376. Santa Barbara: ABC-CLIO.

Arrow, Kenneth J. 1963. Social Choice and Individual Values. 2nd. New Haven: Yale University Press. Ben-Yashar, Ruth, and Sarit Kraus. 2002. "Optimal Collective Dichotomous Choice Under Quota Constraints." Economic Theory 839-852.

Brams, Steven J., and Peter C. Fishburn. 2007. Approval Voting. 2nd. Boston: Springer.

Brams, Steven J., and D. Marc Kilgour. 2014. "When Does Approval Voting Make the "Right Choices"?" In The Mathematics of Decisions, Elections, and Games, edited by Karl-Dieter Crisman and Michael A. Jones, 37-53. Providence: American Mathematical Society.

Brams, Steven J., and Richard F. Potthoff. 2015. "The Paradox of Grading Systems." Public Choice 193210. 
Brams, Steven J., and M. Remzi Sanver. 2010. "Voting Systems that Combine Approval and Preference." In The Mathematics of Preference, Choice, and Order, by Steven J. Brams, William V. Gehrlein and Fred S. Roberts, 215-237. Berlin: Springer-Verlag.

Byron, Michael, ed. 2004. Satisficing and Maximizing. Cambridge: Cambridge University Press.

Dahl, Robert A. 1989. Democracy and Its Critics. New Haven: Yale University Press.

Dasgupta, Partha, and Eric Maskin. 2008. "On the Robustness of Majority Rule." Journal of the European Economic Association 949-973.

Feddersen, Timothy, Sean Gailmard, and Alvaro Sandroni. 2009. "Moral Bias in Large Elections: Theory and Experimental Evidence." American Political Science Review 175-192.

Gigenrenzer, Gerd, Peter M. Todd, and ABC Research Group. 1999. Simple Heuristics That Make Us Smart. New York: Oxford University Press.

Goodin, Robert E., and Christian List. 2006. "A Conditional Defense of Plurality Rule: Generalizing May's Theorem in a Restricted Informational Environment." American Journal of Political Science 940949.

Harsanyi, John C. 1953. "Cardinal Utility in Welfare Economics and in the Theory of Risk-taking ." Journal of Political Economy 434-435.

Inada, Ken-ichi. 1964. "A Note on the Simple Majority Decision Rule." Econometrica 525-531.

List, Christian. 2013. "Social Choice Theory." Vers. Winter 2013 Edition. Stanford Encyclopedia of Philosophy. Edited by Edward N. Zalta. December 18. http://plato.stanford.edu/archives/win2013/entries/social-choice/.

List, Christian, and Robert E. Goodin. 2001. "Epistemic Democracy: Generalizing the Condorcet Jury Theorem." Journal of Political Philosophy 277-306.

Maniquet, Francois, and Philippe Mongin. 2015. "Approval Voting and Arrow's Impossibility Theorem." Social Choice and Welfare 519-532.

May, Kenneth O. 1952. "A Set of Independent Necessary and Sufficient Conditions for Simple Majority Decision." Econometrica 680-684.

Merrill III, Samuel, and Jack Nagel. 1987. "The Effect of Approval Balloting on Strategic Voting under Alternative Decision Rules." American Political Science Review 509-524.

Prasad, Mahendra. 2012. "Condorcet, Preference, and Judgment." 11th Meeting of the Society for Social Choice and Welfare. New Delhi.

Prasad, Mahendra. 2014. "Social Rank Ordering Functions and Arrow's Theorem." 12th Meeting of the Society for Social Choice and Welfare. Boston: Society for Social Choice and Welfare.

Prasad, Mahendra. 2016. "On the Normative Uniqueness of Majority Rule: Generalizing May's Theorem to Arrovian Social Welfare Functions." 74th Annual Conference of the Midwest Political Science Association. Chicago: Midwest Political Science Association.

Rae, Douglas W. 1969. "Decision-Rules and Individual Values in Constitutional Choice." American Political Science Review 40-56.

Riker, William H. 1982. Liberalism Against Populism. Prospect Heights: Waveland Press.

Risse, Mathias. 2004. "Arguing for Majority Rule." Journal of Political Philosophy 41-64.

Rousseau, Jean-Jacques. 1947. The Social Contract. Edited by Charles Frankel. Translated by Charles Frankel. New York: Hafner Publishing Company.

Schwartz, Barry, Andrew Ward, John Monterosso, Sonja Lyubomirsky, Katherine White, and Darrin R. Lehman. 2002. "Maximizing versus satisficing: Happiness is a matter of choice." Journal of Personality and Social Psychology 1178-1197.

Sen, Amartya Kumar. 1970. Collective Choice and Social Welfare. San Francisco: Holden-Day.

Simon, Herbert A. 1955. "A Behavioral Model of Rational Choice." Quarterly Journal of Economics 99118. 
Simon, Herbert A. 1978. "Rationality as a Process and as a Product of Thought." American Economic Review 1-16.

Simon, Herbert A. 1987. Satisficing. Vol. IV, in The New Palgrave Dictionary of Economics, edited by J. Eatwell, M. Milgate and P. Newman, 243-245. London: Macmillan Press.

Taylor, Alan D. 2005. Social Choice and the Mathematics of Manipulation. New York City: Cambridge University Press.

Taylor, Michael. 1969. "Critique and Comment: Proof of a Theorem on Majority Rule." Behavioral Science 228-231.

Young, H. P. 1988. "Condorcet's Theory of Voting." American Political Science Review 1231-1244. 